\section{Management of non-CF bronchiectasis}

\section{P172 TIME TRENDS IN INCIDENCE AND PREVALENCE OF BRONCHIECTASIS IN THE UK}

doi:10.1136/thoraxjnl-2012-202678.233

'JK Quint, 'E Millett, 'JR Hurst, 'L Smeeth, 'J Brown. 'LSHTM, London, UK; ' $U C L$, London, UK

Background The incidence of bronchiectasis in the UK is unknown. No large study has been performed in the UK since the $1950 s^{1}$ and there are few relevant data from non-UK sources. The prevalence is likely to be relatively high as bronchiectasis is a chronic condition, and case ascertainment is increasing with the wider availability of CT scanning. Establishing the healthcare burden is essential for informing allocation of healthcare resources and improving patient experience.

Methods All individuals in the general practise research database (CPRD-GOLD) between $1^{\text {st }}$ January 2004 and 31 ${ }^{\text {st }}$ December 2011 were included. Currently this includes information from 640General Practises in the UK. From this cohort, patients with a diagnosis of bronchiectasis were identified using specific READ codes. We calculated the absolute incidence rates and prevalence of bronchiectasis by calendar year, age and gender.

Results Over the 8 year time period 27,258 individuals $(0.7 \%)$ had a diagnosis of bronchiectasis. The overall incidence increased overtime; 2004 incidence 18/100,000 person years at risk and in 2011 the incidence was $32 / 100,000$ person years at risk. Prevalence also increased year on year, was higher in older age groups ( $>60$ years of age) and was higher in women than in men. The prevalence in 2011 in men and women by age is provided in Table 1 .

Conclusions Bronchiectasis is a relatively common condition in 2011 in the UK, particularly in individuals over the age of 60. Part of the increase in prevalence over time may be due to increasing numbers of CT scans being performed. How the prevalence relates to individual disease burden and health careutilisation is yet to be established.

References

1. Guideline for non-CF Bronchiectasis, British Thoracic Society (July 2010).

Abstract P169 Table 1 Prevalence of bronchiectasis per 100,000 in 2011 in men and women by age

\begin{tabular}{|c|c|c|}
\hline Age Groups (years) & $\begin{array}{l}\text { Prevalence in men per } \\
100,000\end{array}$ & $\begin{array}{l}\text { Prevalence in women per } \\
100,000\end{array}$ \\
\hline$<30$ & 33 & 35 \\
\hline $30-39$ & 55 & 73 \\
\hline $40-49$ & 97 & 146 \\
\hline $50-59$ & 303 & 420 \\
\hline $60-69$ & 639 & 862 \\
\hline $70-79$ & 1089 & 1320 \\
\hline$\geq 80$ & 1101 & 1079 \\
\hline Overall & 227 & 309 \\
\hline
\end{tabular}
IDENTIFIED BY A SPECIALIZED INVESTIGATION PROTOCOL

doi:10.1136/thoraxjnl-2012-202678.234

'A Creamer, ${ }^{2 J}$ Elliot, ${ }^{2} \mathrm{~J}$ Ryan, ${ }^{2 \mathrm{~J}}$ French, ${ }^{2 \mathrm{H}}$ Barker, ${ }^{2} \mathrm{DS}$ Wat, ${ }^{2 \mathrm{H}}$ Baxendale, ${ }^{2} \mathrm{~A}$ Exley, ${ }^{2} \mathrm{CS}$ Haworth, 'RA Floto. 'University of Cambridge, Cambridge, UK; ${ }^{2}$ Papworth Hospital, Cambridge, UK

Rationale Numerous factors have been identified as contributing to the development of bronchiectasis although their relative prevalence remains poorly understood. The Cambridge Centre for Lung Infection (CCRI) at Papworth Hospital has one of the largest specialist bronchiectasis units in Europe. All new patients referred with recurrent or severe chest infections undergo a systematic investigation protocol to determine an underlying cause for their lung disease involving: high-resolution CT (HRCT) scan; full pulmonary function tests; sweat testing and CFTR sequencing; nasal nitric oxide measurements; immunological test including serum immunoglobulins, specific antibody levels pre-and post vaccination, auto-antibody screening; Aspergillus serology and multiple sputum sample testing for conventional and mycobacterial microscopy and culture. We undertook an analysis of the results of these investigations to determine the relative contributions of causal factors in patients with bronchiectasis.

Methods We examined the results and case notes of all 352 patients referred to the CCRI with recurrent chest infections between January 1st 2009 and May 31st 2011. Of these, 202 individuals had HRCT evidence of bronchiectasis. The results of their initial investigations were analysed to determine the proportion of patients we could ascribe a likely cause for their bronchiectasis and whether this affected their subsequent management.

Results Using our investigation strategy, we were able to identify a likely cause in 139/202 (69\%) patients, with 31\% remaining idiopathic. Identifying an underlying cause frequently influenced subsequent patient management. Common causes included post-infection $(22 \%)$, aspiration (7\%), primary and secondary immune-deficiencies (4 and 6\% respectively), and allergic broncho-pulmonary aspergillosis (6\%). 5 new diagnoses of cystic fibrosis were made in patients aged 23, 26, 35, 53 and 64 .

Conclusion This study represents the largest analysis of causative factors of bronchiectasis to date. Using our current investigation algorithm, we can ascribe a cause for bronchiectasis in almost $70 \%$ of new patients. Many of the underlying conditions diagnosed require specialist management.

\section{P174 SEVERITY OF BRONCHIECTASIS ON HIGH RESOLUTION CT SCANNING AND ITS RELATIONSHIP TO CHRONIC BACTERIAL COLONISATION}

doi:10.1136/thoraxjnl-2012-202678.235

'RJ Stretton, 'KE Leighton, ${ }^{2} S$ Marshall, 'S Schembri, 'T Fardon, 'JD Chalmers. 'Department of Respiratory Medicine, Ninewells Hospital, Dundee, Scotland; ${ }^{2}$ Department of Clinical Immunology, Ninewells Hospital, Dundee, Scotland

Introduction Bacterial colonisation and particularly colonisation with Pseudomonas aeruginosa is associated with a more severe clinical course in bronchiectasis. It is often presumed that patients with more extensive radiological bronchiectasis are at higher risk of chronic bacterial colonisation, but data on this is lacking. The aim of this study was to determine if severity of bronchiectasis on HRCT predicts chronic bacterial colonisation.

Methods Data was collected from a specialist bronchiectasis clinic from April 2011 to April 2012. A total of 88 patients were diagnosed with bronchiectasis following assessment by a respiratory physician and a high resolution CT scan. Sputum cultures from the previous 2 years were used to determine colonisation status.

The severity of disease on CT scanning was determined for each patient using a modified Reiff scoring system. This attributed to every lobe a score dependant on the type of Bronchiectasis seen and the number of lobes involved - Cylindrical $=1$, Varicose $=2$, Cystic $=3$. The minimum score is 1 and the maximum score is 18 .

Results 88 patients had bronchiectasis confirmed on HRCT by a clinical radiologist. For each set of patients colonised with a particular organism the average Reiff score was calculated. 11 patients had P. aeruginosa (Reiff mean 5.6, SD 4.8). This score was significantly higher than those patients colonised with other pathogenic microorganisms (Reiff mean 3.4, SD 2.9) ( $p=0.03$ ), and for patients not colonised with microorganisms (Reiff mean 3.0, SD 2.2) ( $p=0.005)$.figure 1 
Among the other organisms isolated 29 patients had Haemophilus influenzae (Reiff Mean 3.7, SD 3.5). This mean Reiff score was significantly lower than for $P$. aeruginosa, $(\mathrm{p}=0.03)$ - figure 1 .

Streptococcus pneumoniae (Reiff Mean 3.4), Moraxella catarrhalis (Reiff Mean 3.7) and Staphylococcus aureus (Reiff Mean 3.2), were also isolated in lesser numbers and a further 28 patients had a mix of other colonising organisms (Reiff Mean 3.5).

Conclusion More severe radiological bronchiectasis is associated with the presence of bacterial colonisation and particularly colonisation with Pseudomonas aeruginosa. This simple assessment of radiological severity may be a useful clinical tool in non-CF bronchiectasis.

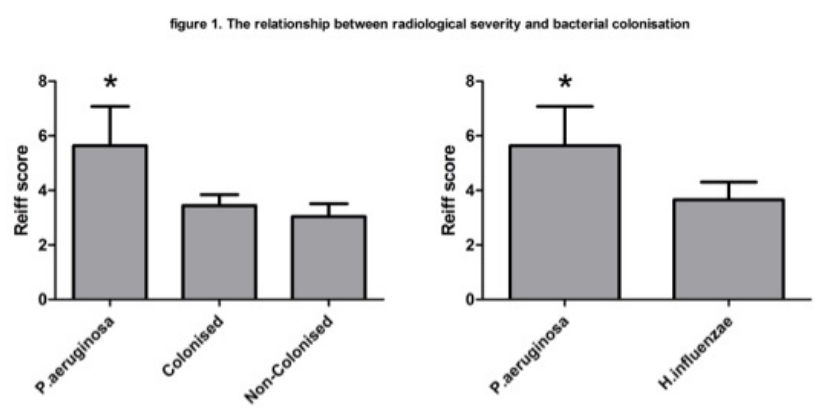

Abstract P174 Figure 1 Radiological severity and bacterial colonisation

\section{P175 THE SHORT TERM VARIABILITY OF SPUTUM MICROBIOLOGY IN NON-CF BRONCHIECTASIS}

doi:10.1136/thoraxjnl-2012-202678.236

MC Coleman, C Hennessy, D Bilton, R Wilson, MR Loebinger. Royal Brompton Hospital, London, UK

Introduction It is recommended that non-CF bronchiectasis patients have sputum cultured annually. This prospective, cohort study is the first to collect monthly cultures to assess this practise by recording the short term variability of sputum bacteriology.

Method 85 patients with non-CF bronchiectasis and daily sputum production were recruited between December 2010 and May 2011. Patients completed daily symptom diaries, and spirometry and sputum samples were collected monthly for 6 months.

Results 58/85 were female, average age 58 (range 17-82). Most common aetiologies were idiopathic $41 / 85$ and post infective 19/85. 64/85 completed follow up.

There were 417 sputum cultures of which only 265 cultured an organism (incl. 130 Pseudomonas aeruginosa (PA), 37 Staphlococcus aureus, 34 Haemophilus influenzae.) 10/64 patients grew no organism throughout the study despite monthly samples. 30/64 had one or more positive culture for PA, including 6 patients with first isolates. Of those with positive cultures, 28/64 patients grew the same organism in all positive cultures (15/64 grew PA only) whereas $26 / 64$ had variability in the microbe isolated. A total of 37 exacerbations occurred at time of clinic visit. Of these 4/37 were associated with growth of a pathogen not previously isolated in this patient. However, 20/37 sputum samples at the time of clinical exacerbation showed no growth despite no prior antibiotic use. 22 patients used prophylactic antibiotics of whom 14 grew PA and 2 grew no organism.

122 infective exacerbations resulted in the use of 145 antibiotics courses. 14/64 had no exacerbations over 6 months. 9/64 had one and 41/64 had two or more (median 2). Spirometry and symptom scores remained relatively stable throughout the 6 months and were not significantly different in patients with $\geq 2$ exacerbations.

Conclusion This study demonstrates the limitations of annual sputum cultures. There was significant microbe variability and importantly a significant number of first PA isolations over a short follow-up period. Furthermore, a large percentage of patients had no microbe isolated (including at exacerbation) suggesting a possible use of future molecular microbe techniques.

\section{P176 COPD-RELATED BRONCHIECTASIS; A REAL CLINICAL ENTITY WITH IMPACT ON DISEASE COURSE AND OUTCOMES}

doi:10.1136/thoraxjnl-2012-202678.237

'EH Baker, ${ }^{1} \mathrm{~N}$ Kumar, ${ }^{2} \mathrm{D}$ Lai, ${ }^{1} \mathrm{~B}$ Sansom, ${ }^{1} \mathrm{~A}$ Nair, ${ }^{1} \mathrm{~V}$ Vlahos. ${ }^{1}$ St George's University of London, London, UK; ${ }^{2}$ Chelsea and Westminster Hospital, London, UK

Introduction and Objectives Non-CF bronchiectasis is defined as 'symptoms of persistent or recurrent bronchial sepsis related to irreversibly damaged and dilated bronchi' [BTS guidelines 2010]. Radiographic evidence of 'damaged and dilated bronchi' can be seen on CT Thorax in up to $50 \%$ of COPD patients. However the contribution of radiographic bronchiectasis to the clinical course of COPD is not fully understood. We aimed to determine the impact of bronchiectasis on lung function, sputum microbiology and outcomes in COPD patients, independent of coexisting emphysema and bronchial wall thickening (BWT).

Methods COPD patients admitted with first exacerbation 1998-2008 were identified retrospectively using ICD10 codes J44.0,1,8,9. Patients with high resolution CT images within 2 years of admission were included. CT scans were graded by consensus of 2 senior thoracic radiologists for severity of bronchiectasis, emphysema and BWT on a 5 point scale (0-absent, 1-minor, 2-mild, 3-moderate, 4-severe). Operational definitions were set prior to scan review and radiologists were blinded to clinical parameters.

Results 406 patients ( $71 \pm 11$ years, $56 \%$ male, $\mathrm{FEV}_{1} 52 \pm 23 \%$ predicted) were included. 278 (69\%) patients had bronchiectasis: minor, 112 (40\%); mild, 81 (29\%); moderate, 62 (22\%); severe 23 (8\%). There was considerable overlap between bronchiectasis and other pathologies (figure). Bronchiectasis severity correlated with severity of BWT $(r=0.276, p<0.001)$ and emphysema $(r=0.120$, $p=0.015$ ). After adjustment for severity of emphysema and BWT, increasing severity bronchiectasis was not an independent predictor of lung function parameters, but independently determined isolation of Pseudomonas aeruginosa (Odds ratio (OR) 1.39 (95\% CI 1.07$1.80), p=0.013)$ and atypical mycobacteria from sputum cultures (OR 2.44 (95\% CI 1.04-5.69), p=0.040). After correction for increasing severity emphysema, BWT, age, gender and comorbidities, increasing severity bronchiectasis determined annual admissions (regression coefficient $\mathrm{B}=0.14$ (95\% CI $0.00-0.28), \mathrm{p}=0.044$ ) and inpatient days $(B=2.1$ (95\% CI $0.8-3.4), p=0.001)$ for respiratory causes, but did not influence survival from first hospital admission $(p=0.257)$.

Conclusions Radiographic bronchiectasis in COPD patients is associated with increased respiratory infection and hospitalisation, independent of coexisting emphysema and BWT. COPD-related bronchiectasis is therefore a diagnosis with important clinical implications. Further research should determine whether treatment strategies for non-CF bronchiectasis can improve the clinical course of COPD-related bronchiectasis.

\section{P177 OUTCOMES OF PSEUDOMONAS AERUGINOSA (PA) ERADICATION IN NON-CYSTIC FIBROSIS BRONCHIECTASIS. FORCED VITAL CAPACITY (FVC) AND LATENT PERIOD FROM GROWTH TO ERADICATION ARE SIGNIFICANT VARIABLES IN ERADICATION SUCCESS}

doi:10.1136/thoraxjnl-2012-202678.238

S Ejiofor, G Packer, K Mckinley, J Whitehouse. Birmingham Heartlands Hospital-Heart of England NHS Trust, Birmingham, England 